\title{
COMPARAÇÃO ENTRE AS SOLUÇÕES ORAIS DE MANITOL A10\% E BIFOSFATO DE SÓDIO NO PREPARO MECÂNICO DO CÓLON
}

\author{
COMPARISON BETWEEN SODIUM BIPHOSPHATE AND 10\% ORAL MANNITOL \\ SOLUTIONS FOR MECHANICAL BOWEL PREPARATION
}

\author{
Marssoni Deconto Rossoni ${ }^{1}$; Maria Cristina Sartor ${ }^{2}$; \\ Andrea Maciel de Oliveira Rossoni ${ }^{3}$; Renato de Araujo Bonardi ${ }^{4}$; \\ Zacarias Alves de Souza Filho,EBC-PR ${ }^{4}$
}

\begin{abstract}
RESUMO: Objetivo: Comparar o uso das soluções orais de manitol a 10\% e de bifosfato de sódio no preparo mecânico do cólon quanto a qualidade da limpeza, a tolerabilidade e as alterações hidroeletrolíticas e da osmolaridade plasmática. Método: Foram analisados 60 pacientes de modo randomizado, duplo-cego e prospectivo, com indicação de colonoscopia. A qualidade da limpeza intestinal foi analisada pelo examinador através da classificação de Beck A tolerabilidade à ingestão baseou-se na pesquisa do gosto, presença ou não de desconforto, aparecimento de efeitos adversos e a quantidade da solução ingerida. Foram dosados o sódio, potássio, cálcio, magnésio, fósforo, uréia, creatinina, glicose, hematócrito, hemoglobina e calculado a osmolaridade plasmática, antes e após a ingestão da solução oral de preparo inestinal. Resultados: Ambas as soluções atingiram qualidade de preparo classificado como bom ou superior em mais de $80 \%$ dos pacientes. O uso do bifosfato de sódio determinou menor desconforto e melhor tolerância, apesar de não ter sido superior ao manitol quanto à análise do gosto e presença de efeitos adversos. O bifosfato induziu ao aumento e o manitol a uma redução da osmolaridade, reflexo do que ocorreu com o sódio plasmático nos dois grupos respectivamente. O bifosfato ainda determinou alteração significativa dos níveis séricos de fósforo, cálcio, magnésio e potássio, sem repercussões clínicas. Conclusão: Ambos os tipos de preparo intestinal determinaram qualidade de limpeza adequada. O bifosfato de sódio, apesar de melhor tolerado, determina maior quantidade de alterações hidroeletrolíticas (Rev. Col. Bras. Cir. 2008; 35(5): 323-328).
\end{abstract}

Descritores: Colo; Infecções bacterianas; Manitol/administração \& dosagem; Fosfatos.

\section{INTRODUÇÃO}

O preparo mecânico do cólon, apesar de dispensado por alguns autores para determinadas operações colorretais, continua sendo de fundamental importância em vários métodos diagnósticos e terapêuticos nas doenças colorretais. Durante a colonoscopia, método fundamental na investigação das doenças colorretais, especialmente no rastreamento do câncer colorretal, é essencial que a limpeza intestinal seja de alta qualidade ${ }^{1}$. Durante operações sobre o cólon, o preparo mecânico prévio pode permitir a detecção de lesões não diagnosticadas previamente com potencial de influenciar e modificar os procedimentos per-operatórios ${ }^{2}$.

Outro aspecto importante a ser considerado é a tolerabilidade do paciente em relação ao tipo de preparo intestinal empregado. $\mathrm{O}$ preparo adequado não deve proporcionar desconforto para o paciente ou, ao menos, que este seja mínimo, associado à menor taxa de efeitos adversos.

O preparo intestinal ideal, não foi estabelecido. Sabese que cada método usado oferece vantagens e desvanta- gens, tanto para os pacientes quanto para os profissionais médicos envolvidos ${ }^{3,4}$. Atualmente, um dos métodos mais utilizados para o preparo do cólon é o uso oral da solução de manitol à $10 \%$.

Em virtude da ocorrência de casos de desidratação subseqüente ao preparo com manitol a $10 \%$, e de explosões durante a eletrocauterização em colonoscopias e operações, devido à proliferação bacteriana e distensão colônica decorrentes da fermentação do manitol por bactérias intestinais produtoras de hidrogênio e metano, outros protocolos de preparo têm sido estudados ${ }^{5-13}$.

Foi demonstrado que a administração oral de solução salina de bifosfato de sódio pode proporcionar boa qualidade de limpeza intestinal com a necessidade de ingestão de pequeno volume da solução, o que é habitualmente bem tolerado pelo paciente ${ }^{2,13-15}$

Este estudo foi desenvolvido com o objetivo de comparar os preparos mecânicos do cólon realizados com a solução oral de manitol a $10 \%$ e a de bifosfato de sódio, quanto à qualidade de limpeza intestinal; a tolerabilidade do paciente

\footnotetext{
1. Aluno do Programa de Pós-Graduação em Clínica Cirúrgica da UFPR

2. Doutora em Cirurgia pela Faculdade de Medicina da Universidade de São Paulo. Médica da Unidade de Coloproctologia do Hospital de Clínicas da UFPR.

3. Mestre em Medicina Interna pela UFPR.

4. Professor do Departamento de Cirurgia da UFPR.

Recebido em 08/04/2008

Aceito para publicação 08/07/2008

Conflito de interesses: nenhum

Fonte de financiamento: nenhuma

Trabalho realizado no Serviço de Cirurgia Geral da Unidade de Coloproctologia do Hospital de Clínicas da UFPR.

Este trabalho representa parte integrante da dissertação de Mestrado do aluno Marssoni Deconto Rossoni no programa de Pós-Graduação em Clínica Cirúrgica da Universidade Federal do Paraná (UFPR).
} 
para a ingestão das soluções de preparo e as repercussões hidroeletrolíticas associadas ao uso dos mesmos.

\section{MÉTODOS}

Este estudo foi aprovado pelo Comitê de Ética em Pesquisa em Seres Humanos do Hospital de Clínicas da UFPR.

Sessenta pacientes submetidos consecutivamente a avaliação colonoscópica eletiva pelas indicações listadas na tabela 1, no Serviço de Endoscopia do Hospital de Clínicas da Universidade Federal do Paraná (UFPR), foram randomizados por sorteio para um dos dois métodos de preparo intestinal, manitol ou bifosfato de sódio, constituindo-se ao final da randomização dois grupos de 30 pacientes.

O preparo intestinal iniciou-se na véspera do exame com uso de dieta sem resíduos. No dia do procedimento, os pacientes foram esclarecidos a respeito da finalidade do estudo, sendo obtido o consentimento para realização do mesmo. Os pacientes ingeriram no local do exame uma das soluções de preparo oral: manitol a 10\%, em solução de $1000 \mathrm{ml}$ (500 ml de manitol a $20 \%$ diluídos em $500 \mathrm{ml}$ de água filtrada) ou bifosfato de sódio em solução de $150 \mathrm{ml}$, composto por $9 \mathrm{~g}$ de fosfato de sódio dibásico e $36 \mathrm{~g}$ de fosfato de sódio monobásico, diluídos em água destilada. Esta segunda solução foi aromatizada com oito gotas de essência de abacaxi, oito gotas de essência de baunilha e adocicada com oito go-

Tabela 1 - Indicações para colonoscopia.

\begin{tabular}{lcc}
\hline Indicação & Total $(\mathbf{n = 6 0 )}$ & $\mathbf{\%}$ \\
\hline Diarréia crônica & 10 & 16,7 \\
Hematoquezia & 5 & 8,3 \\
Dor abdominal & 5 & 8,3 \\
Doença inflamatória intestinal & 9 & 15 \\
Seguimento de neoplasia operada & 9 & 15 \\
Polipose colônica familial & 13 & 21,7 \\
Neoplasia colorretal & 2 & 3,3 \\
Sangue oculto positivo nas fezes & 1 & 1,7 \\
Seguimento pós polipectomia & 6 & 10 \\
Total & 60 & 100 \\
\hline
\end{tabular}

tas de sacarina). Os pacientes foram orientados previamente, por meio de texto explicativo, quanto aos cuidados dietéticos para a obtenção do preparo adequado. Nenhum paciente recebeu qualquer tipo de reposição endovenosa durante o preparo. Todos ingeriram um litro de água até, no máximo, duas horas antes da realização do exame

$\mathrm{O}$ pesquisador desconhecia as características do paciente e a suspeita diagnóstica até a realização do questionário de pesquisa, que foi aplicado após a determinação do tipo de solução. Todas as endoscopias foram realizadas por um único examinador, que desconhecia o preparo intestinal empregado em cada caso.

Foram excluídos do protocolo de estudo, pacientes com as seguintes características: gestantes, obstrução intestinal, emergências cirúrgicas ou clínicas, insuficiência renal, insuficiência hepática e falha na execução do preparo dietético ou laxativo.

Foram analisados, para os dois grupos de estudo, três classes de parâmetros: a)Qualidade da limpeza intestinal; b)Tolerabilidade do preparo; c)Alterações hidroeletrolíticas e da osmolaridade plasmática.

Qualidade da Limpeza Intestinal

Ao término de cada colonoscopia, o examinador classificou a qualidade do preparo do cólon encontrada durante o exame de acordo com a classificação de $\operatorname{Beck}^{16}$ (Tabela 2).

Tolerabilidade do Preparo Intestinal

A tolerabilidade à ingestão das soluções de preparo intestinal baseou-se nas informações quanto aos seguintes ítens:

a) gosto da solução ingerida, classificado em ótimo, bom ou ruim;

b) desconforto para ingerir a solução, considerado como presente ou ausente;

c) efeitos adversos: náuseas, vômitos e cólicas abdominais, associados ou não;

d) quantidade de solução ingerida, classificada em boa tolerância, tolerância moderada e intolerável; Plasmática

Alterações Hidroeletrolíticas e da Osmolaridade

Imediatamente antes do início da ingestão da solução oral de preparo intestinal e imediatamente antes do término do exame, foram colhidas de cada paciente amostras de

Tabela 2 - Classificação da qualidade de limpeza do cólon segundo Beck ${ }^{16}$.

\begin{tabular}{ll}
\hline Categorias & Descrição \\
\hline Excelente & $\begin{array}{l}\text { Avaliação integral do relevo mucoso, sem dificuldade técnica. Conteúdo intraluminal constituído por líquido } \\
\text { transparente ou translúcido, sem grumos ou resíduos. }\end{array}$
\end{tabular}

Boa

Avaliação integral do relevo mucoso, sem dificuldade. Conteúdo intraluminal constituído por líquido transparente, com grumos de muco ou pequenas partículas, facilmente aspiráveis.

Regular Avaliação integral do relevo mucoso, com pequena dificuldade técnica. Conteúdo intraluminal constituído por suco entérico turvo e aderente à mucosa ou pequenos resíduos fecais, com possibilidade de aspiração após lavagem com água.

Ruim Avaliação parcial do relevo mucoso. Conteúdo intraluminal constituído por fezes líquidas e/ou pastosas, impossíveis de serem completamente aspiradas, mesmo após lavagem com água. 
sangue para dosagem sérica de sódio, potássio, cálcio, magnésio, fósforo, uréia, creatinina e glicose que foram mensuradas por meio de Analisador Bioquímico Automatizado Mega (BAYERÒ). A hemoglobina e o hematócrito foram mensurados pelo método de contagem de partículas (contador de células COULTERÒ STKS Analyzer). A osmolaridade sérica foi deduzida a partir da dosagem do sódio, glicose e uréia séricos, utilizando-se a seguinte fórmula ${ }^{17,18}$ :

Osmolaridade $=2 \mathrm{xNa}+[$ Glicose $/ 18]+[$ Uréia/5,6]

Além dos parâmetros acima as seguintes variáveis foram registradas e analisadas: a) Tempo inicial de ação da solução: Intervalo de tempo em minutos entre a ingestão da primeira fração da solução e a hora da primeira evacuação; b) Frequiência evacuatória: Número total de evacuações desde a ingestão da primeira fração de solução até o início do exame endoscópico; c) Tempo total de ação da solução: Intervalo de tempo em minutos entre a primeira e a última evacuação; d) Formação de bolhas de ar na luz do cólon.

Tratamento Estatístico

Para a análise estatística das variáveis independentes utilizaram-se os testes não-paramétricos "Comparação entre duas Proporções" e "Mann-Whitney", por meio do software "Primer of Biostatistics - versão 4.0", e os testes "Qui-Quadrado com correção de Yates" e "Exato de Fisher", por meio do software "Epi-Info, versão 6.04b (1997)".

Para o tratamento estatístico das amostras relacionadas ou pareadas, foram utilizados os testes paramétrico "t de Student pareado" e o não paramétrico "Wilcoxon", por meio do software "Primer of Biostatistics - versão 4.0 (1997)".

O nível de significância adotado foi de $5 \%$.

\section{RESULTADOS}

No grupo manitol, $16(53,3 \%)$ eram do sexo masculino e 14 do feminino. A mediana de idade foi de 46 anos, variando entre 11 a 80 anos. No grupo bifosfato de sódio, 12 (40\%) pacientes eram do sexo masculino e 18 do sexo feminino. A mediana de idade foi de 42 anos, variando entre 16 a 90 anos. Na comparação dos dados gerais, a idade $(\mathrm{p}=0,745)$ e o sexo $(\mathrm{p}=0,4376)$ não apresentam diferença estatística entre os grupos de estudo.

Não houve nenhum tipo de complicação durante e após a colonoscopia.

Qualidade da Limpeza Intestinal:

Quanto à qualidade do preparo, os resultados encontrados nos dois grupos estão mostrados na Tabela 2.
Houve predominância significativa de preparos classificados como de qualidade excelente no grupo bifosfato $(\mathrm{p}=0,0379)$. Porém, quando consideramos as duas classes de resultados favoráveis em conjunto (excelente + boa) não há diferença estatística entre os dois grupos. $\mathrm{O}$ mesmo acontece com a comparação entre os grupos na classe de preparo classificados como regular e ruim.

Tolerabilidade do Preparo Intestinal

Os resultados quanto a tolerabilidade do preparo intestinal nos dois grupos estão apresentados na tabela 3.

Não houve diferença estatística entre os grupos de estudo quanto ao gosto da solução ingerida. Os dois tipos de preparo também não mostram diferenças quanto a apresentação de efeitos adversos (náuseas, vômitos e cólicas). Entretanto, os pacientes que ingeriram bifosfato como preparo, referiram menos desconforto à ingestão e melhor tolerância quando comparados ao grupo manitol.

Alterações Hidroeletrolíticas e da Osmolaridade Plasmática

Quanto aos valores obtidos dos exames laboratoriais, no pré e pós-preparo, e do cálculo da osmolaridade plasmática antes e após o preparo, a tabela 4 mostra os resultados obtidos.

Pacientes do grupo de preparo com manitol mostraram alterações estatisticamente significativas no sódio sérico, na uréia e na osmolaridade, enquanto que pacientes preparados com bifosfato alteraram significativamente as dosagens séricas de sódio, potássio, cálcio, magnésio, fósforo e osmolaridade plasmática.

Enquanto o preparo com manitol mostrou queda na taxa de sódio associada à baixa da osmolaridade plasmática, o preparo com bifosfato se comportou de maneira oposta, elevando a taxa de sódio e determinando incremento da osmolaridade plasmática.

O tempo inicial de ação da solução apresentou mediana de 50 minutos em ambos grupos. No grupo manitol a variação foi de 20 a 140 minutos. No grupo bifosfato de sódio foi de 25 a 150 minutos ( $\mathrm{p}=0,661)$.

A frequência evacuatória foi, em média, 7,1 vezes para o grupo manitol, com mediana de 7,0, variando de 1,0 a 16 vezes. Para o grupo bifosfato de sódio, a média foi 6,0 vezes, mediana de 5,0, com variação de 3 a 14 vezes. Não houve diferença estatística.

O tempo total de ação da solução no grupo manitol foi, em média, de 139,6 minutos, variando de 70 a 195 minutos. O tempo total de ação da solução bifosfato de sódio foi de 144,3 minutos, variando de 65 a 260 minutos $(\mathrm{p}=0,679)$.

Tabela 3 - Resultados sobre a qualidade do preparo intestinal.

\begin{tabular}{lccc}
\hline Classificação do Preparo & Grupo Manitol(n) & Grupo Bifosfato(n) & P \\
\hline Excelente & 12 & 21 & 0,0379 \\
Boa & 13 & 5 & NS \\
Regular & 4 & 4 & NS \\
Ruim & 1 & 0 & NS \\
Total & 30 & 30 & \\
\hline
\end{tabular}

NS = não significativo. 
A formação de bolhas intracolônicas, observada durante a colonoscopia, ocorreu em $5(16,7 \%)$ pacientes, apenas no grupo bifosfato de sódio $(\mathrm{p}=0,0261)$.

\section{DISCUSSÃo}

O preparo intestinal adequado é condição indispensável e determinante de resultados, quer seja durante colonoscopia ou mesmo durante operações colorretais.

Nenhum método de limpeza intestinal é agradável, mas a aceitação pelos pacientes aumentou depois do aparecimento dos métodos orais ${ }^{19}$.

No presente estudo comparou-se a qualidade e tolerabilidade, bem como as alterações hidroeletrolíticas induzidas pelo preparo intestinal utilizando-se as soluções orais de bifosfato de sódio ou manitol à $10 \%$. A frequiência de exames com qualidade de preparo excelente ou boa foi de $83,4 \%$ para o grupo manitol e de $86,7 \%$ para o grupo bifosfato de sódio (Tabela 3). Apesar do bifosfato ter número superior de preparos classificados como excelente segundo Beck ${ }^{16}$, as diferenças existentes entre as classes "excelente e boa" é muito pequena, uma vez que em ambas as classes deve ser possível a avaliação integral do relevo mucoso, não existir qualquer dificuldade técnica e o conteúdo intestinal ser líquido e transparente (Tabela 2). Além disso, neste estudo, o tipo de preparo não teve influência sobre os limites proximais alcançados pelo colonoscópio durante o exame.

A formação de bolhas de espuma intracolônica ocorreu exclusivamente no grupo do bifosfato de sódio, abrangendo $16,7 \%$ dos exames $(\mathrm{p}=0,026)$. Existe relato do uso concomitante de bifosfato de sódio e simeticona com o objetivo de diminuir a formação de bolhas, melhorando o exame do cólon $^{20}$. Nesse estudo, a formação de bolhas não prejudicou a qualidade final do exame.

Quanto à análise da tolerabilidade, no presente estudo houve o cuidado metodológico de não associar-se, no protocolo de preparo intestinal de ambos os grupos, medicações antieméticas ou quaisquer outras substâncias que pudessem alterar os resultados.

O uso de drogas antieméticas tem sido preconizado no controle dos efeitos indesejados durante o preparo ${ }^{21}$.

Em ambos os grupos ocorreram mais de $50 \%$ de efeitos clínicos adversos (Tabela 4). Apesar de a náusea ter sido a queixa mais freqüente no grupo bifosfato de sódio e a cólica a mais freqüente no grupo manitol, não houve diferença estatística entre os dois grupos quanto ao aparecimento de efeitos adversos $(\mathrm{p}=0,6005)$.

A aceitabilidade do manitol pelo paciente, em vários estudos, seja por volume ou por efeitos colaterais como náuseas e vômitos, pode ser melhorada com o uso associado de antieméticos ou fracionamento do volume com ingestão lenta ${ }^{12}$.

Em estudo comparativo com 220 pacientes que receberam manitol a $10 \%$ ou bifosfato de sódio, as queixas de cólicas e sensação de plenitude foram mais freqüentes para aqueles que ingeriram a solução de manitol, embora sem significância estatística. No mesmo estudo, em relação ao paladar das soluções, o manitol a $10 \%$ foi preferido pelos pacientes ${ }^{2}$.

A opinião do paciente quanto ao gosto da solução não mostrou diferença estatística significativa entre os dois grupos. Este fato pode ser devido à adição de adoçante e essências aromáticas na solução de bifosfato de sódio, o qual normalmente é referido nos trabalhos científicos como de sabor ruim.

No presente trabalho, ao contrário da maioria dos estudos disponíveis na literatura pertinente, procedeu-se a uma análise criteriosa e pormenorizada das alterações de eletrólitos e de osmolaridade plasmática capazes de ocorrer pelo preparo específico de cada solução oral utilizada. Para isto, houve a necessidade de não incluir nos protocolos de preparo, qualquer re-hidratação venosa após o início do preparo. Neste sentido, foi uniformizado o aporte oral de $1000 \mathrm{ml}$ de água, para todos os pacientes do estudo, até no máximo, duas horas antes da realização do exame.

Os resultados obtidos em relação ao sódio plasmático, e conseqüentemente, em relação à osmolaridade plasmática,

Tabela 4 - Resultados da tolerabilidade do preparo intestinal.

\begin{tabular}{|c|c|c|c|}
\hline Parâmetros de tolerabilidade & Grupo Manitol (n) & Grupo Bifosfato (n) & $\mathbf{P}$ \\
\hline \multicolumn{4}{|l|}{ A) Gosto da solução ingerida } \\
\hline Ótimo & 0 & 1 & NS \\
\hline Bom & 19 & 22 & NS \\
\hline Ruim & 11 & 7 & NS \\
\hline \multicolumn{4}{|l|}{ B) Desconforto a ingestão } \\
\hline Presente & 16 & 3 & 0,0009 \\
\hline \multicolumn{4}{|l|}{ C) Efeitos adversos } \\
\hline Náuseas & 14 & 19 & NS \\
\hline Vômitos & 07 & 06 & NS \\
\hline Cólicas & 10 & 07 & NS \\
\hline Total de pacientes & 16 & 19 & NS \\
\hline \multicolumn{4}{|c|}{ D) Quantidade da solução ingerida } \\
\hline Boa tolerância & 15 & 30 & 0,0003 \\
\hline Tolerância moderada & 14 & 0 & 0,0003 \\
\hline Intolerável & 1 & 0 & NS \\
\hline
\end{tabular}

$N S=$ não significativo . 
foram caracteristicamente oposto (Tabela 5). Enquanto o preparo com manitol produziu redução significativa do nível plasmático de sódio, este se elevou significativamente no grupo que usou bifosfato, compreensivamente, uma vez que este sal usado é sódico. Isto determinou aumento significativo na osmolaridade plasmática no grupo bifosfato e o inverso no grupo manitol. Tal achado, apesar de não ter desencadeado repercussão clínica na presente série, deve, definitivamente ser levado em consideração na escolha do tipo de solução oral e protocolo de preparo intestinal a ser empregado nas variadas situações onde se impõe o preparo mecânico colônico.

Além disso, o uso de bifosfato de sódio como solução oral de preparo intestinal, determinou, na presente casuística, elevações significativas do nível sérico de fósforo, situação inerente à sua fórmula, e reduções significativas das taxas de potássio, cálcio e magnésio (Tabela 5).

Outros relatos na literatura em relação a solução oral de bifosfato de sódio, descrevem aumento significativo nos níveis séricos de sódio e transitório no de fosfato, com diminuição nos de cálcio e potássio ${ }^{22,23}$. Já, para o manitol é descrito alterações nos níveis do sódio e potássio séricos, com desidratação, elevando o hematócrito ${ }^{24,25}$.

Em situações especiais, como em pacientes portadores de insuficiência renal, insuficiência cardíaca congestiva, cirrose hepática com ascite ou os de labilidade eletrolítica comprovada, a ocorrência de alterações hidroeletrolíticas deve ser levada em consideração quanto ao risco de descompensação clínica no paciente que for submetido a preparo com bifosfato de sódio ${ }^{2,13,26-28}$. Quanto ao manitol, o maior cuidado é reservado aos pacientes idosos, nos quais a desidratação e a hiponatremia podem não ser compensadas pelos mecanismos homeostáticos.

Há relatos na literatura que associam úlceras aftóides ao uso do bifosfato de sódio ${ }^{29,30}$. Neste estudo não se observou lesões macroscópicas na mucosa colônica decorrentes seja do bifosfato de sódio, seja do manitol.

O estudo permitiu concluir que a solução oral salina de bifosfato é melhor tolerada que a solução de manitol a $10 \%$ com qualidade de preparo adequado. Entretanto, existiu maior número significativo de distúrbios hidroeletrolíticos com o uso de bifosfato de sódio.

$\mathrm{Na}$ falta de um protocolo de preparo intestinal ideal, acreditamos que seja benéfico individualizar, de acordo com a situação clínica e indicações, a utilização das soluções orais disponíveis para uso, uma vez que, como comprovada pelo presente estudo, as alterações e conseqüentes repercussões podem variar significativamente de acordo com o protocolo e solução oral escolhida para o preparo intestinal.

Tabela 5 - Alterações hidroeletrolíticas e da osmolaridade plasmática.

\begin{tabular}{|c|c|c|c|c|c|c|}
\hline \multirow{3}{*}{$\begin{array}{l}\text { Dosagens } \\
\text { Sódio }\end{array}$} & \multicolumn{3}{|c|}{ Grupo Manitol } & \multicolumn{3}{|c|}{ Grupo Bifosfato } \\
\hline & Antes & Depois & \multirow{2}{*}{$\frac{\mathbf{p}}{<0,0001}$} & Antes & Depois & \multirow{2}{*}{$\frac{\mathbf{P}}{0,0001}$} \\
\hline & $137,4 \pm 2,1$ & $135,3 \pm 2,3$ & & $137,6 \pm 2,5$ & $139,7 \pm 2,6$ & \\
\hline Potássio & $4,2 \pm 0,5$ & $4,0 \pm \quad 0,5$ & NS & $4,4 \pm \quad 0,4$ & $4,0 \pm 0,4$ & $<0,0001$ \\
\hline Cálcio & $9,1 \pm 0,5$ & $8,8 \pm 1,1$ & NS & $9,0 \pm 0,7$ & $8,6 \pm 0,7$ & 0,006 \\
\hline Magnésio & $2,0 \pm 0,2$ & $2,0 \pm \quad 0,2$ & NS & $2,1 \pm 0,3$ & $2,0 \pm 0,2$ & 0,047 \\
\hline Fósforo & $3,4 \pm \quad 1,0$ & $3,8 \pm \quad 1,1$ & NS & $3,2 \pm 0,7$ & $7,2 \pm 1,7$ & $<0,0001$ \\
\hline Uréia & $26,6 \pm 7,8$ & $24,5 \pm \quad 7,8$ & 0,003 & $26,7 \pm 7,8$ & $26,8 \pm 7,2$ & NS \\
\hline Creatinina & $0,8 \pm 0,1$ & $0,7 \pm \quad 0,2$ & NS & $0,7 \pm 0,1$ & $0,7 \pm 0,2$ & NS \\
\hline Glicose & $101,3 \pm 19,1$ & $106 \pm 22,6$ & NS & $98,3 \pm 12,2$ & $109 \pm 33,7$ & NS \\
\hline Hemoglobina & $13,4 \pm 1,0$ & $13,4 \pm \quad 1,1$ & NS & $13,5 \pm 2,1$ & $13,4 \pm 2,4$ & NS \\
\hline Hematócrito & $39,8 \pm 3,1$ & $40,1 \pm 3,2$ & NS & $40,2 \pm 5,9$ & $39,6 \pm 6,6$ & NS \\
\hline Osmolaridade & $285,2 \pm 4,5$ & $280,8 \pm \quad 4,5$ & $<0,0001$ & $285,5 \pm 5,0$ & $290,3 \pm 5,9$ & $<0,0001$ \\
\hline
\end{tabular}

$N S=$ não significativo

\begin{abstract}
Background: To compare the use of sodium biphosphate and $10 \%$ mannitol solutions for mechanical bowel preparation in terms of cleansing quality, tolerability, disorder in water and electrolyte balance, and plasma osmolality. Method: Sixty patients who had been referred for colonoscopy were analyzed in a randomized, double-blind, prospective study. The quality of bowel cleansing was analyzed by the examiner using Beck's classification. Ingestion tolerability was established by investigating taste, whether the patient felt any discomfort or not, the development of adverse effects and the amount of solution ingested. The following measurements were made before and after ingestion of the oral bowel preparation solution: sodium, potassium, calcium, magnesium, phosphorus, urea, creatinine, glucose, hematocrit, hemoglobin and plasma osmolality. Results: Both solutions resulted in bowel preparations that were classified as good or superior in over $80 \%$ of the patients. The use of sodium biphosphate resulted in less discomfort and better tolerance, although it was not superior to mannitol in terms of taste or the presence of adverse effects. Sodium biphosphate led to an increase, and mannitol to a decrease, in osmolality, reflecting the changes in plasma sodium in both groups. The former also resulted in a significant measurement change in serum phosphorus, calcium, magnesium and potassium levels, without any clinical repercussions. Conclusion: Both types of bowel preparation resulted in adequate cleansing. Sodium biphosphate, although better tolerated, leads to more alterations in water and electrolyte balance.
\end{abstract}

Key words: Colon; Bacterial Infections; Mannitol/administration \& dosage; Phosphates. 


\section{REFERÊNCIAS}

1. Adler M, Quenon M, Even-Adin D, Jeanmart J, Van Gossum A, Bourgeois N, Cremer M. Whole gut lavage for colonoscopy: a comparison between two solutions. Gastrointest Endosc. 1984; 30(2):65-7.

2. Oliveira LCC, Zuccaro AM. Estudo prospectivo e randomizado comparando solução de fosfato de sódio oral e manitol para o preparo de cólon para colonoscopia. Rev Col Brasil Cir. 1999; 26(3):335-8.

3. Young CJ, Simpson RR, King DW, Lubowski DZ. Oral sodium phosphate solution is a superior colonoscopy preparation to polyethylene glycol with bisacodyl. Dis Colon Rectum. 2000; 43(11):1568-71.

4. Thomson J, Phull P. Audit of bowel preparation with Picolax (sodium picosulfate plus magnesium citrate) for colonoscopy. Int J Clin Pract. 2006; 60(5):602-3.

5. Ell C, Fischbach W, Keller R, Dehe M, Mayer G, Schneider B, Albrecht U, Schuette W; Hintertux Study Group. A randomized, blinded, prospective trial to compare the safety and efficacy of three bowel-cleansing solutions for colonoscopy.Endoscopy. 2003; 35(4):300-4.

6. Kastenberg D, Barish C, Burack H, Dalke Dd, Duckor S, Putnam W, Valenzuela G. Tolerability and patient acceptance of sodium phosphate tablets compared with 4-L PEG solution in colon cleansing results of 2 identically designed, controlled, parallel group, multicenter phase 3 trials. J Clin Gastroenterol. 2007; 41(1):54-61.

7. Hookey LC, Depew WT, Vanner SJ. A prospective randomized trial comparing low-dose oral sodium phosphate plus stimulant laxatives with large volume polyethylene glycol solution for colon cleansing. Am J Gastroenterol. 2004; 99(11):2217-22.

8. Davis GR, Santa Ana CA, Morawski SG, Fordtran JS. Inhibition of water and electrolyte absorption by polyethylene glycol (PGE). Gastroenterology. 1980; 79(1):35-9.

9. Keighley MRB, Taylor EW, Hares MM, Arabi Y, Youngs D, Bentley S, Burdon DW. Influence of oral mannitol bowel preparation on colonic microflora and the risk of explosion during endoscopic diathermy. Br J Surg. 1981; 68(5):554-6.

10. Zanoni CE, Bergamini C, Bertoncini M, Bertoncini L, Garbini A. Whole-gut lavage for surgery: a case of intraoperative colonic explosion after administration of mannitol. Dis. Colon Rectum. 1982; 25(6):580-1.

11. Ambrose NS, Johnson M, Burdon DW, Keighley MRB. A physiological appraisal of polyethylene glycol and a balanced electrolyte solution as bowel preparation. Br J Surg. 1983; 70(4):428-30.

12. Habr-Gama A, Vieira MJF, Alves PA, Souza Jr. AHS, Sototuka JK, Gama-Rodrigues JJ, Travassos VHCR. Preparo do cólon para colonoscopia. Estudo prospectivo randomizado com solução de manitol a $10 \%$ e com solução eletrolítica contendo polietilenoglicol. GED. 1986; 5(4):127-32.

13. Habr-Gama A, Kiss DR, Araujo SE, Bringel RWA. Preparo intestinal para cirurgia colorretal eletiva: polietilenoglicol (PEG) $\mathrm{x}$ fosfato de sódio (FS) - Resultados de estudo prospectivo e randomizado. Rev Bras Coloproct. 1998; 18(2):85-9.

14. Handelsman JC, Zeiler S, Coleman J, Dooley, W, Walrath JM. Experience with ambulatory preoperative bowel preparation at the Johns Hopkins Hospital. Arch Surg. 1993; 128(4):441-4.

15. Tjandra JJ, Chan M, Tagkalidis PP. Oral sodium phosphate (Fleet) is a superior colonoscopy preparation to Picopre (sodium picosulfatebased preparation). Dis Colon Rectum. 2006; 49(5):616-20.

16. Beck DE, Harford FJ, Dipalma JA. Comparison of cleansing methods in preparation for colonic surgery. Dis Colon Rectum. 1985; 28(3):491-5.
17. Ziochevsky ERM, Paes LSN, Imperial MCG, Rios, MBS. Índices, fórmulas, tabelas, gráficos e bulários. In: Matsumoto T, Carvalho WB, Hirschheimer MR. Terapia Intensiva Pediátrica. $2^{\text {a }}$ ed. São Paulo: Atheneu; 1997. p. 1205-1318.

18. Humphreys MH. Fluid \& Electrolyte Management. In: Way LW. Surgical diagnosis \& treatment. $10^{\text {th }}$ ed. East Norwalk: Lange; 1994. p. 129-142.

19. Rostom A, Jolicoeur E, Dubé C, Grégoire S, Patel D, Saloojee N, Lowe C. .A randomized prospective trial comparing different regimens of oral sodium phosphate and polyethylene glycolbased lavage solution in the preparation of patients for colonoscopy. Gastrointest Endosc. 2006; 64(4):544-52.

20. Sudduth RH, Sherman KE, Deangelis S, Mcnally PR. The effectiveness of simethicone in improving visibility during colonoscopy when given with a sodium phosphate solution: a double-blind randomized study. Gastrointest Endosc. 1995; 42(5):413-6.

21. Rhodes JB, Engstrom J, Stone KFM. Metoclopramide reduces the distress associated with colon cleansing by on oral electrolyte overload. Gastrointest Endosc. 1978; 24(4):162-3.

22. Vanner SJ, Macdonald PH, Paterson WG, Prentice RS, Costa LR, Beck IT. A randomized prospective trial comparing oral sodium phosphate with standart polyethylene glycol-based lavage solution (Golytely) in the preparation of patients for colonoscopy. Am J Gastroenterol. 1990; 85(4):422-7.

23. Rhodes JB, Zuangulis JE, Williams CH, Gonzales G, Moffat RE. Oral electrolyte overload to cleanse the colon for colonoscopy. Gastrointest Endosc.1977;24(1):24-6.

24. Nahas SC. Colonoscopia. Método diagnóstico e terapêutico. Rev Hosp Clin Fac Med São Paulo. 1995; 50(3):154-9.

25. Resende MS, Guimarães M, Oliveira EJM, Araújo JJ. Colonoscopia: contribuição no diagnóstico e tratamento das doenças do cólon. Rev Bras Coloproct. 1987; 7(2):52-5.

26. Barkun A, Chiba N, Enns R, , Natsheh S, Pham C, Sadowski D, Vanner S. Commonly used preparations for colonoscopy: efficacy, tolerability, and safety-a Canadian Association of Gastroenterology position paper. Can J Gastroenterol. 2006; 20(11):699-710.

27. Hwang KL, Chen WT, Hsiao KH, Chen HC, Huang TM, Chiu CM, Hsu GH. Prospective randomized comparison of oral sodium phosphate and polyethylene glycol lavage for colonoscopy preparation. World J Gastroenterol. 2005; 11(47):7486-93.

28. Rex DK. Dosing considerations in the use of sodium phosphate bowel preparations for colonoscopy. Ann Pharmacother. 2007; 41(9):1466-75.

29. Hixson LJ. Colorectal ulcers associated with sodium phosphate catharsis. Gastrointest. Endosc. 1995; 42(1):101-2.

30. Zwas FR, Cirillo NW, El-Serag HB, Eisen RN. Colonic mucosal abnormalities associated with oral sodium phosphate solution. Gastrointest Endosc. 1996; 43(5):463-6.

\section{Como citar este artigo:}

Rossoni MD, Sartor MC, Souza Filho ZA, Bonardi RA, Rossoni AM. Comparação entre as soluções orais de manitol a $10 \%$ e bifosfato de sódio no preparo mecânico do cólon. Rev Col Bras Cir. [periódico na Internet] 2008; 35(5). Disponível em URL: http://www.scielo.br/ rcbc

Endereço para correspondência: Dr. Marssoni Deconto Rossoni Rua Pedro Nogarolli, 277 80710-050 - Curitiba - PR E-mail:marssoni@gmail.com 